The Structure of the 1,1,2,2Tetranitroethanediide Anion BERNT KLEWE

Universitetets Kjemiske Institutt, Blindern, Oslo 3, Norway

Tnfrared spectra of 29 1,1-dinitrocarbanion salts ${ }^{1}$ and Raman spectra of potas. sium dinitromethanide and the deuterated analogue ${ }^{2}$ have been reported. An essentially planar arrangement of the $-\mathrm{C}\left(\mathrm{NO}_{2}\right)_{2}{ }^{-}$ group has been found in the crystal structure of potassium 4,4-dinitrobutenamide $^{3}$ with a distance of only $2.51 \AA$ between two nitro group oxygen atoms. A similar arrangement has been observed for the dinitrocyanomethanide anion in a two-dimensional X-ray study of $\mathrm{RbC}\left(\mathrm{NO}_{2}\right)_{2} \mathrm{CN}$. $^{4}$ The geometry of the $-\mathrm{C}\left(\mathrm{NO}_{2}\right)_{2}{ }^{-}$group may be quite sensitive to the steric requirements of the third group linked to the carbon atom; in the crystal structure of hydrazonium trinitromethanide ${ }^{5}$ the two independent anions present are both found to be propeller shaped with two nitro groups twisted less than $10^{\circ}$, whereas the third is twisted $41^{\circ}$ and $74^{\circ}$, respectively, from a planar configuration. The crystal structure investigation of $\mathrm{CaC}\left[\mathrm{C}(\mathrm{CN})_{2}\right]_{3} \cdot 6 \mathrm{H}_{2} \mathrm{O}^{\circ}$ shows that in this case the $\mathrm{C}\left[\mathrm{C}(\mathrm{CN})_{2}\right]_{3}{ }^{2-}$ anion, which has geometrical requirements similar to that of $\mathrm{C}\left(\mathrm{NO}_{2}\right)_{3}^{-}$, is found to be a symmetrical propeller with a twist angle of $24^{\circ}$.
Two-dimensional crystallographic investigations of the potassium and rubidium salts of 1,1,2,2-tetranitroethane have been carried out. The potassium salt was synthezised according to Hunter ${ }^{7}$ and the rubidium compound was obtained in a metathetic reaction with $\mathrm{RbCl}$ in aqueous solution. Because of the instability of the crystals all X-ray diagrams were recorded at $-20^{\circ} \mathrm{C}$.

$\mathrm{K}_{2} \mathrm{C}_{2} \mathrm{~N}_{4} \mathrm{O}_{8}$ crystals are monoclinic, space group $C 2 / c$ or $C c$. The unit cell, containing 4 formula units, has the following parameters: $a=13.0_{3} \AA, b=7.5_{6} \AA, c=9.0_{0} \AA$, $\beta=106 .{ }^{\circ}$. The structure was determined by Fourier methods in the $h 0 l$ projection assuming the space group to be $C 2 / c$ and refined by the minimum residual method. ${ }^{8}$ This space group requires the anion to possess a twofold axis of symmetry or a centre of symmetry. The latter requirement would force the nitro groups to be highly twisted to avoid too short oxygen-oxygen contacts, whereas an ideal model having $D_{2}$ symmetry with planar or propeller shaped $-\mathrm{C}\left(\mathrm{NO}_{2}\right)_{2}^{-}$groups twisted about the carbon-carbon bond seemed favourable. The final Fourier projection along the $b$ axis with the anions indicated is shown in Fig. 1. The $y$-param. eters were established by independent translation of cation and anion along the $b$ axis in the $h k 0$ projection, which shows considerable overlapping. The $R$-value obtained for the $171 h 0 l$ and $h k 0$ reflections (28 absences included with $F=F_{\min } / 2$ ) by the minimum residual method was $11 \%$.

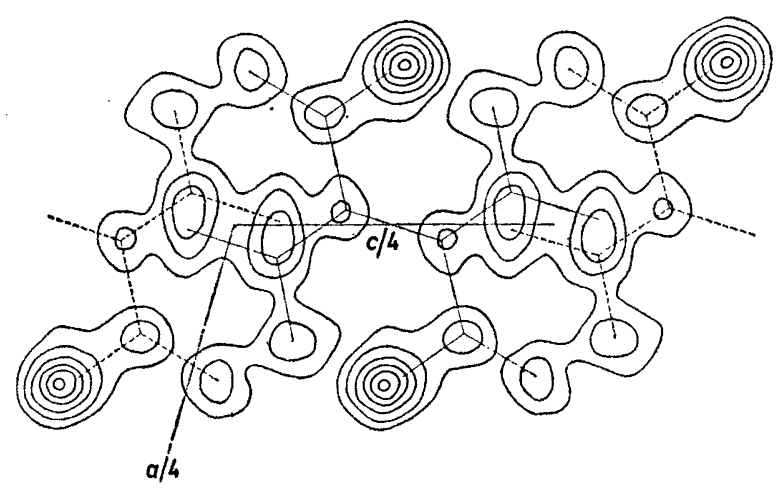

Fig. 1. Fourier projection of $\mathrm{K}_{2} \mathrm{C}_{2} \mathrm{~N}_{4} \mathrm{O}_{8}$ along the $b$ axis. 


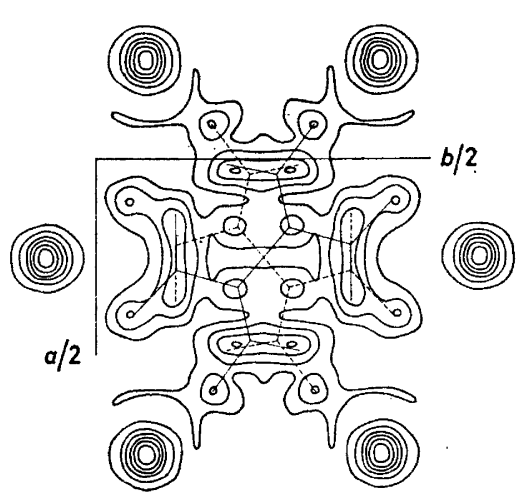

Fig. 2. Fourier projection of $\mathrm{Rb}_{2} \mathrm{C}_{2} \mathrm{~N}_{4} \mathrm{O}_{8}$ along the $c$ axis.

$\mathrm{Rb}_{2} \mathrm{C}_{2} \mathrm{~N}_{4} \mathrm{O}_{8}$ crystals are orthorhombic with $a=7.6_{8} \AA, b=13.3_{0} \AA, c=8.8_{3} \AA$, and 4 formula units in the unit cell. The space group Pccn requires the same symmetry of the anion as for the potassium salt. The structure was determined in the $h k 0$ projection by Fourier methods and the minimum residual method. The final Fourier map is shown in Fig. 2. The rubidium atoms seem to overlap com. pletely. The $z$-parameters were determined from the $0 k l$ projection. The final $R$-value for $166 h k 0$ and $0 k l$ reflections $(34$ accidental extinctions included) was $11 \%$.

The coordination number of the cation is six and eight for the potassium and the rubidium salt, respectively. In both structures the crystallographic twofold axis is normal to the carbon-carbon bond. According to the large twist angles of the approximately planar $-\mathrm{C}\left(\mathrm{NO}_{2}\right)_{2}{ }^{-}$ groups, the dihedral $\mathrm{N}-\mathrm{C}-\mathrm{C}-\mathrm{N}$ angles being about $60^{\circ}$ and $.70^{\circ}$, respectively, the double bond character of the $\mathrm{C}-\mathrm{C}$ bond must be negligible. There are indications of propeller twists of the nitro groups. Differences in coordination of the oxygen atoms may lead to different twist angles, thus destroying the $D_{2}$ symmetry. A deviation from this symmetry cannot, however, be stated with certainty because of the considerable overlap of atoms in the projections.

A three-dimensional investigation of these salts is now being undertaken.
1. Kamlet, M.J., Oesterling, R.E. and Adolph, H. G. J. Chem. Soc. 19655838.

2. Lippincott, E. R., Kenney, T. E., Nanney, T. R. and Weiffenbach, C. K. J. Chem. Soc. B 196732.

3. Holden, J. R. and Dickinson, C. W. Am. Cryst. Ass. Winter Meeting, 1967, Program and Abstracts; J. Am. Chem. Soc. In press.

4. Bjornstad, H. J. and Klewe, B. To be published.

5. Dickens, B. Chem. Commun. 1967246.

6. Bekoe, D. A., Gantzel, P. K. and Trueblood, K. N. Acta Cryst. 22 (1967) 657.

7. Hunter, L. J. Chem. Soc. 123 (1923) 547.

8. Bhuiya, A. K. and Stanley, E. Acta Cryst. 16 (1963) 981.

Received July 1, 1967.

\section{Fluorimetric Determination of Boron with Chinizarin in Concentrated Sulfuric Acid}

\section{A. HOLME}

\section{University of Oslo, Chemical Institute A, Blindern, Oslo 3, Norway}

$D^{1}$ uring a systematic investigation of the complex formation in concentrated sulfuric acid between boric acid and a series of hydroxyanthraquinones (1,4dihydroxy-, 1,5-dihydroxy-, 1,8-dihydroxy-, 1,2,3-trihydroxy-, 1,2,4-trihydrexy-, and 1,2,3,5,6,7-hexahydroxyanthraquinone), it was found that chinizarin (1,4-dihydroxyanthraquinone) was the only reagent producing a fluorescent complex. The present paper describes the fluorimetric determination of boron with chinizarin.

Previous studies on the fluorescence of boron complexes in concentrated sulfuric acid have been published by Marcantona. tos, Marcantonatos and Monnier. ${ }^{1}$ The basis of fluorospectrophotometry is the principle that in dilute solutions and at a given wavelength the fluorescence intensity is proportional with the concentration of the fluorescent substance. ${ }^{2},{ }^{3}$

Experimental. Reagents. Chinizarin (K \& K Laboratories, USA) was dissolved in concentrated sulfuric acid, precipitated in and 